CERN-TH $/ 2002-056$

LAPTH-906/02

\title{
Conformally coupled supermultiplets in four and five dimensions
}

\author{
Sergio Ferrara' and Emery Sokatchevf \\ CERN Theoretical Division, CH 1211 Geneva 23, Switzerland
}

\begin{abstract}
We obtain by superfield methods the exceptional representations of the $\operatorname{OSp}(2 N / 4, \mathbb{R})$ and $\mathrm{SU}(2,2 / 1)$ superalgebras which extend to supersingletons of $\mathrm{SU}(2,2 / 2 N)$ and $\mathrm{F}(4)$, respectively. These representations describe superconformally coupled multiplets and appear in three- and four-dimensional superconformal field theories which are holographic descriptions of certain antide Sitter supergravities.
\end{abstract}

\footnotetext{
${ }^{1}$ Laboratori Nazionali di Frascati, INFN, Italy

${ }^{2}$ On leave of absence from Laboratoire d'Annecy-le-Vieux de Physique Théorique LAPTH, B.P. 110, F-74941 Annecy-le-Vieux et l'Université de Savoie
} 


\section{Introduction}

In the early days of investigations by physicists [1] of the unitary irreducible representations (UIRs) of $\mathrm{SO}(d, 2)$ (the $d$-dimensional conformal group), it was remarked that there exist special degenerate representations ("singletons") which give rise to just a pair of UIRs when reduced to the subgroup $\mathrm{SO}(d-1,2)$.

These pairs of modules are "shadow pairs" [2], i.e., they have the same Casimir eigenvalues, and correspond to the so-called "conformally coupled fields" [1] in $d$ dimensions. We recall that $\mathrm{SO}(d, 2)$ can be viewed either as the isometry group of $\mathrm{AdS}_{d+1}$ or as the conformal group in $d$ dimensional Minkowski space $\mathrm{M}_{d}$. The two are related by the fact that a certain compactification of $\mathrm{M}_{d}$ can be regarded as the boundary of $\operatorname{AdS}_{d+1}$.

In the present note we extend this analysis to superconformal symmetry. We show that four-(or five-)dimensional supersingleton multiplets are decomposed in an analogous way into pairs of three-(or four-)dimensional "mirror" supermultiplets corresponding to the superalgebra embedding $\operatorname{OSp}(2 N / 4, \mathbb{R}) \subset \mathrm{SU}(2,2 / 2 N)$ (or $\mathrm{SU}(2,2 / 1) \subset \mathrm{F}(4))$. A similar result does not hold in the six-dimensional case because the superalgebra $\mathrm{F}(4)$ is not a subalgebra of $\operatorname{OSp}\left(8^{*} / 4\right)$.

These exceptional representations appeared recently in the physics literature in different contexts. In the four-dimensional case (with $N=4$ ) it is the study of the AdS/CFT correspondence in the presence of "defects" [3, 国]. In the five-dimensional case, in IIB supergravity on $\mathrm{AdS}_{5} \times \mathrm{T}^{1,1}$ the lowest-dimensional chiral primary operator $\operatorname{Tr}(A B)$ describes precisely a conformally coupled scalar, which means that it has an extension (together with its shadow) to a supersingleton representation of $\mathrm{F}(4)$ [5].

The paper is organized as follows. In Section 2 we explain how two scalar UIRs of the $d$ dimensional conformal group $\mathrm{SO}(\mathrm{d}, 2)$ of shadow conformal weights $\ell_{ \pm}=(d \pm 1) / 2$ merge together to form a scalar singleton UIR of the $d+1$-dimensional conformal group $\mathrm{SO}(\mathrm{d}+1,2)$. In Section 3 we examine the possibilities to embed a $d$-dimensional conformal superalgebra in a $d+1$ dimensional one. We show that $\operatorname{OSp}(N / 4, \mathbb{R}) \subset \mathrm{SU}(2,2 / N)$ and $\mathrm{SU}(2,2 / 1) \subset \mathrm{F}(4)$, but $\mathrm{F}(4)$ $\not \subset \operatorname{OSp}\left(8^{*} / 4\right)$. Next, in Section 4 we study the branching of $d=4$ scalar supersingletons into pairs of $d=3$ supermultiplets, corresponding to the embeddings $\operatorname{OSp}(1 / 4, \mathbb{R}) \subset \mathrm{SU}(2,2 / 1)$ and $\operatorname{OSp}(2 N / 4, \mathbb{R}) \subset \mathrm{SU}(2,2 / 2 N)$. We show that the two $d=3$ "shadow" scalars obtained by breaking up the $d=4$ singleton, are found in two "mirror" $d=3$ supermultiplets of the same conformal dimension 2 , but belonging to two complementary $\mathrm{R}$ symmetry irreps (selfdual and anti-self-dual tensors of $\mathrm{SO}(2 \mathrm{~N}))$. The $d=3$ multiplets obtained can be viewed as "supercurrents", i.e., as squares of the two types of $d=3$ supersingletons. In Section 5 we perform a similar reduction from $d=5$ to $d=4$, corresponding to the embedding $\mathrm{SU}(2,2 / 1) \subset$ $\mathrm{F}(4)$. We show that the $d=5$ supersingleton branches into a pair of $d=4$ chiral supermultiplets with "anomalous" dimension $3 / 2$.

\section{Relationship between scalar UIRs of $\mathrm{SO}(d, 2)$ and $\mathrm{SO}(d+1,2)$}

In this section, following Fronsdal [1], we explain the basic phenomenon which underlies this work. The question we want to answer is how to upgrade a scalar UIR of the conformal group in $d$ dimensions $\mathrm{SO}(d, 2)$ to a UIR of the conformal group in $d+1$ dimensions $\mathrm{SO}(d+1,2)$.

\footnotetext{
${ }^{1}$ The same question can be asked about higher spin representations 1], but scalars are sufficient for our purpose.
} 
The answer is very simple: We have to take two UIRs of $\mathrm{SO}(d, 2)$, namely, scalars of conformal dimension $\ell_{1}=(d-1) / 2$ and $\ell_{2}=(d+1) / 2$; together they form a massless scalar UIR of $\mathrm{SO}(d+1,2)$ (or a "singleton" in the AdS sense [6]) of canonical conformal dimension $(d-2) / 2$. The explanation follows.

The algebra of $\mathrm{SO}(d, 2)$ contains $\mathrm{SO}(d-1,1)$ (Lorentz) generators $M_{m n}=-M_{n m}$, translations $P_{m}$, boosts $K_{m}$ and dilatation $D$. Here $m=0,1, \ldots, d-1$ is a vector index of $\mathrm{SO}(d-1,1)$. The commutation relations relevant to our discussion are

$$
\left[P_{m}, K_{n}\right]=i M_{m n}+i \eta_{m n} D, \quad\left[D, P_{m}\right]=i P_{m}, \quad\left[D, K_{m}\right]=-i K_{m}
$$

The group $\mathrm{SO}(d, 2)$ is non-compact, so its UIRs are infinite-dimensional Hilbert spaces. To construct such a space, consider a ground state $|\ell, 0\rangle$ of conformal dimension $\ell$ and spin 0 (i.e., a singlet of the Lorentz subgroup $\mathrm{SO}(d-1,1))$ defined by

$$
M_{m n}|\ell, 0\rangle=K_{m}|\ell, 0\rangle=0, \quad D|\ell, 0\rangle=i \ell|\ell, 0\rangle .
$$

Here the boosts $K_{m}$ play the rôle of annihilators whereas the translations $P_{m}$ are the creators. Applying the latter to the ground state, we obtain the Hilbert space

$$
\mathcal{H}_{\ell, 0}: \quad|\ell, 0\rangle, P_{m}|\ell, 0\rangle, P_{m} P_{n}|\ell, 0\rangle, \ldots
$$

In it we find states with spin 0 obtained by acting with the scalar $P^{m} P_{m}$ :

$$
|\ell+2 k, 0\rangle=\left(P^{m} P_{m}\right)^{k}|\ell, 0\rangle .
$$

Note the absence of states $|\ell+2 k+1,0\rangle$ in $\mathcal{H}_{\ell, 0}$.

Now, we wish to upgrade this $\mathrm{SO}(d, 2)$ UIR to a UIR of $\mathrm{SO}(d+1,2)$. The algebra of $\mathrm{SO}(d+1,2)$ contains $d$ additional Lorentz generators $M_{m d}$, one boost (annihilator) $K_{d}$ and one translation (creator) $P_{d}$. If $|\ell, 0\rangle$ is to become the ground state of the $\mathrm{SO}(d+1,2)$ UIR with spin 0 (a singlet of $\mathrm{SO}(d, 1))$ that we want to build up, then it must be annihilated by $M_{m d}$ and $K_{d}$ :

$$
M_{m d}|\ell, 0\rangle=K_{d}|\ell, 0\rangle=0 .
$$

Further, the new creator $P_{d}$ applied to this ground state produces the state

$$
|\ell+1,0\rangle=P_{d}|\ell, 0\rangle,
$$

which is not present in $\mathcal{H}_{\ell, 0}$ (3). We must regard this state as the ground state of a new $\mathrm{SO}(d, 2)$ Hilbert space $\mathcal{H}_{\ell+1,0}$. At the same time, it should be viewed as an excitation of the $\mathrm{SO}(d+1,2)$ ground state. This explains why we need two UIRs of $\mathrm{SO}(d, 2)$ to form one UIR of $\mathrm{SO}(d+1,2)$.

Next, applying $P_{d}$ twice we create the state $|\ell+2,0\rangle=\left(P_{d}\right)^{2}|\ell, 0\rangle$. This time, a similar state is contained in $\mathcal{H}_{\ell, 0}$ (3), namely $P^{m} P_{m}|\ell, 0\rangle$. Irreducibility then leads to the identification of these two states:

$$
P^{m} P_{m}|\ell, 0\rangle=\left(P_{d}\right)^{2}|\ell, 0\rangle \Leftrightarrow P^{\mu} P_{\mu}|\ell, 0\rangle=0, \quad \mu=0,1, \ldots, d .
$$

Thus, in $d+1$ dimensions we are dealing with a massless UIR of the conformal group $\mathrm{SO}(d+1,2)$ (or a "singleton" in the AdS terminology). 
Finally, the identification condition (77) should be compatible with the action of all the annihilators $K_{\mu}$ of $\mathrm{SO}(d+1,2)$. This leads to the well-known result that the conformal dimension must take the canonical value for a $d+1$-dimensional massless scalar,

$$
P^{\mu} P_{\mu}|\ell, 0\rangle=0 \Rightarrow \ell=\frac{(d+1)-2}{2}=\frac{d-1}{2} .
$$

In summary, the two $\mathrm{SO}(d, 2)$ ground states $|(d-1) / 2,0\rangle$ and $|(d+1) / 2,0\rangle$ create two Hilbert spaces $\mathcal{H}_{(d-1) / 2,0}$ and $\mathcal{H}_{(d+1) / 2,0}$ which merge into the single Hilbert space $\mathcal{H}_{(d-1) / 2,0}^{\prime}$ of an $\mathrm{SO}(d+1,2)$ massless UIR of canonical dimension $\ell=(d-1) / 2$ and Lorentz spin 0.

Note that $\ell_{ \pm}=(d \pm 1) / 2$ are the "shadow dimensions" [2] corresponding to the same value of the quadratic Casimir of $\mathrm{SO}(d, 2)$ (or "AdS mass" [07, 8, 9])

$$
C_{2}=M^{2}=\ell(\ell-d)=\frac{1}{4}\left(1-d^{2}\right) .
$$

\section{Embedding of superconformal algebras in diverse dimensions}

In the preceding section we have seen that two shadow scalars in $d$ dimensions combine to form a singleton (massless) scalar in $d+1$ dimensions. In the supersymmetric case we would expect to find a similar relationship between scalar supersingletons in $d+1$ dimensions (massless scalar multiplets of the $N$-extended superconformal algebra $\mathcal{A}_{d+1}^{N}$ ) and pairs of scalar multiplets of the superconformal algebra $\mathcal{A}_{d}^{N}$ in $d$ dimensions. An obvious necessary condition for this to take place is the existence of the embedding $\mathcal{A}_{d}^{N} \subset \mathcal{A}_{d+1}^{N}$.

In this section we discuss the structure of the superconformal algebras $\operatorname{OSp}(N / 4, \mathbb{R})(d=3)$, $\mathrm{SU}(2,2 / N)(d=4), \mathrm{F}(4)(d=5)$ and $\operatorname{OSp}\left(8^{*} / 2 N\right)(d=6)$ 10, 11. We show that the embeddings $\operatorname{OSp}(N / 4, \mathbb{R}) \subset \mathrm{SU}(2,2 / N)(d=3 \leftarrow d=4)$ [12] and $\mathrm{SU}(2,2 / 1) \subset \mathrm{F}(4) \quad(d=4 \leftarrow d=5)$ exist, but $\mathrm{F}(4) \subset \mathrm{OSp}\left(8^{*} / 4\right)(d=5 \leftarrow d=6)$ is not possible.

To do this, we examine the structure of the anticommutator of the odd generators case by case, starting from the highest dimension $d=6$ for which a standard superconformal algebra exists 13.

The $d=6$ superalgebra $\operatorname{OSp}\left(8^{*} / 2 N\right)$ has the even part $\operatorname{SO}(6,2) \times \operatorname{USp}(2 N)$. Correspondingly, the odd generators $\Sigma_{\alpha}^{a}$ carry two indices. The index $\alpha=1, \ldots, 8$ is in one of the two inequivalent spinor irreps of $\mathrm{SO}(6,2)$ (a non-compact form of $\mathrm{SO}(8)$ ), e.g., $\boldsymbol{8}_{s}$. The index $a=1, \ldots, 2 N$ is in the fundamental irrep of $\operatorname{USp}(2 N)$. These generators satisfy the pseudoreality condition

$$
\Sigma_{\alpha}^{a}=C_{\alpha \beta} \Omega^{a b}\left(\Sigma_{\beta}^{b}\right)^{*},
$$

where $C_{\alpha \beta}$ is the charge conjugation matrix and $\Omega^{a b}=-\Omega^{b a}$ is the $\operatorname{USp}(2 N)$ symplectic matrix. The anticommutator of two odd generators has the following structure:

$$
\left\{\Sigma_{\alpha}^{a}, \Sigma_{\beta}^{b}\right\}=\Omega^{a b} \gamma_{\alpha \beta}^{\mu \nu} M_{\mu \nu}+\mathbb{I}_{\alpha \beta} T^{a b} .
$$

Here $M_{\mu \nu}=-M_{\nu \mu}$ are the generators of $\mathrm{SO}(6,2), \mathbb{I}_{\alpha \beta}$ is the identity matrix in the appropriate Majorana-Weyl basis and $T^{a b}=T^{b a}$ are the generators of $\operatorname{USp}(2 N)$. We also note the decomposition of a $8 \times 8$ matrix $A_{\alpha \beta}$ in terms of antisymmetrized products of gamma matrices:

$$
A_{\alpha \beta}=\mathbb{I}_{(\alpha \beta)} A^{1}+\gamma_{[\alpha \beta]}^{\mu \nu} A_{\mu \nu}^{28}+\gamma_{(\alpha \beta)}^{\mu \nu \lambda} A_{\mu \nu \lambda}^{35}
$$


Here we have indicated the symmetry properties of the gamma matrix products, as well as the dimension of each $\mathrm{SO}(6,2)$ irrep.

Now, consider the unique $d=5$ superconformal algebra $\mathrm{F}(4)$ [14] whose even part is $\mathrm{SO}(5,2) \times$ $\mathrm{USp}(2)$. The odd generators and their anticommutator have the same form as in the case $d=6$, $\alpha=1, \ldots, 8$ being an index of the unique spinor representation of $\mathrm{SO}(5,2)$ (a non-compact form of $\mathrm{SO}(7))$ and $a=1,2$ being a $\mathrm{USp}(2) \sim \mathrm{SU}(2)$ index. What changes, however, is the decomposition (11):

$$
A_{\alpha \beta}=\mathbb{I}_{(\alpha \beta)} A^{1}+\gamma_{[\alpha \beta]}^{\mu} A_{\mu}^{7}+\gamma_{[\alpha \beta]}^{\mu \nu} A_{\mu \nu}^{21}+\gamma_{(\alpha \beta)}^{\mu \nu \lambda} A_{\mu \nu \lambda}^{35} .
$$

We see that the $\mathbf{2 8}$ of $\mathrm{SO}(6,2)$ splits into a $\mathbf{7}$ and a $\mathbf{2 1}$ of $\mathrm{SO}(5,2)$, both being antisymmetric matrices. Consequently, trying to break up the $\operatorname{OSp}\left(8^{*} / 4\right)$ anticommutator (10) so that the corresponding $\mathrm{F}(4)$ relation would emerge, we encounter a problem: It is not possible to keep only the 21 generators of $\mathrm{SO}(5,2)$ without the extra 7 generators of $\mathrm{SO}(6,2)$. We conclude that the embedding $\mathrm{F}(4) \subset \mathrm{OSp}\left(8^{*} / 4\right)$ does not exist.

The next step is to move from $d=5$ to $d=4$. The $d=4$ superconformal algebra $\mathrm{SU}(2,2 / N)$ has the even part $\mathrm{SO}(4,2) \times(\mathrm{S}) \mathrm{U}(N)$ (in general, the R symmetry group is $\mathrm{U}(N)$, except for $N=4$ where it is $\mathrm{SU}(4)$ ). This time the odd generators $\Sigma_{\alpha}^{a}$ (and their conjugates $\bar{\Sigma}_{a}^{\alpha}$ ) carry indices $\alpha=1, \ldots, 4$ and $a=1, \ldots, N$ in the fundamental irreps of $\mathrm{SU}(2,2) \sim \mathrm{SO}(4,2)$ and of $\mathrm{SU}(N)$, respectively:

$$
\left\{\Sigma_{\alpha}^{a}, \bar{\Sigma}_{b}^{\beta}\right\}=\delta_{a}^{b}\left(\gamma^{\mu \nu}\right)_{\alpha}^{\beta} M_{\mu \nu}+\delta_{\alpha}^{\beta} T_{b}^{a} .
$$

The relevant matrix decomposition now concerns two kinds of matrices:

$$
A_{\alpha \beta}=\gamma_{[\alpha \beta]}^{\mu} A_{\mu}^{6}+\gamma_{(\alpha \beta)}^{\mu \nu \lambda} A_{\mu \nu \lambda}^{10}, \quad A_{\alpha}^{\beta}=\delta_{\alpha}^{\beta} A^{1}+\left(\gamma^{\mu \nu}\right)_{\alpha}^{\beta} A_{\mu \nu}^{15},
$$

where $\left(\gamma^{\mu \nu}\right)_{\alpha}^{\beta}$ is traceless. Let us now try to reduce $\mathrm{F}(4)$ to $\mathrm{SU}(2,2 / 1)$ (we set $N=1$ in order to match the numbers of odd generators). To this end we have to break up the $d=5$ generators $\Sigma_{\alpha}^{a}$ into a pair of $d=4$ generators $\Sigma_{\alpha}$ and $\bar{\Sigma}^{\beta}$ and then try to fit the pieces of the generators of the even part. We note that the $\mathbf{2 1}$ of $\mathrm{SO}(5,2)$ decomposes into $\mathbf{1 5}$ and $\mathbf{6}$ of $\mathrm{SO}(4,2)$. According to (14), the symmetry property of the $\mathbf{6}$ does not match that of the $d=4$ anticommutator $\left\{\Sigma_{\alpha}, \Sigma_{\beta}\right\}$, so we have to set the latter to zero. Further, there is room for the $\mathbf{1 5}$ in the $d=4$ anticommutator $\left\{\Sigma_{\alpha}, \bar{\Sigma}^{\beta}\right\}$. Thus, we arrive at the $\mathrm{SU}(2,2 / 1)$ algebra:

$$
\left\{\Sigma_{\alpha}, \Sigma_{\beta}\right\}=0, \quad\left\{\Sigma_{\alpha}, \bar{\Sigma}^{\beta}\right\}=\left(\gamma^{\mu \nu}\right)_{\alpha}^{\beta} M_{\mu \nu}+\delta_{\alpha}^{\beta} T .
$$

We conclude that the embedding $\mathrm{SU}(2,2 / 1) \subset \mathrm{F}(4)$ is possible.

Finally, consider the reduction from $d=4$ to $d=3$. The superalgebra $\operatorname{OSp}(N / 4, \mathbb{R})$ has the even part $\mathrm{SO}(3,2) \times \mathrm{SO}(N)$. The odd generators $\Sigma_{\alpha}^{a}$ carry indices $\alpha=1, \ldots, 4$ in the spinor irrep of $\mathrm{Sp}(4, \mathbb{R}) \sim \mathrm{SO}(3,2)$ and $a=1, \ldots, N$ in the vector irrep of $\mathrm{SO}(N)$. In addition, they satisfy the reality condition $\Sigma_{\alpha}^{a}=\Omega_{\alpha \beta} \bar{\Sigma}^{a \beta}$. Their anticommutator is

$$
\left\{\Sigma_{\alpha}^{a}, \Sigma_{\beta}^{b}\right\}=\delta^{a b}\left(\gamma^{\mu \nu}\right)_{\alpha \beta} M_{\mu \nu}+\Omega_{\alpha \beta} T^{a b},
$$

where $T^{a b}=-T^{b a}$ are the $\mathrm{SO}(N)$ generators. Note also the matrix decomposition

$$
A_{\alpha \beta}=\Omega_{\alpha \beta} A^{1}+\gamma_{[\alpha \beta]}^{\mu} A_{\mu}^{5}+\gamma_{(\alpha \beta)}^{\mu \nu} A_{\mu \nu}^{10},
$$

where $\gamma_{[\alpha \beta]}^{\mu}$ is traceless, $\Omega^{\alpha \beta} \gamma_{[\alpha \beta]}^{\mu}=0$. Now we can see how the reduction from $\operatorname{SU}(2,2 / N)$ works: The $d=3$ odd generators are the real parts of the $d=4$ ones; $\mathrm{U}(N)$ becomes $\mathrm{SO}(N) ; \mathrm{SU}(2,2)$ becomes $\operatorname{Sp}(4, \mathbb{R})$. At the last step the $\mathbf{1 5}$ of $\mathrm{SU}(2,2)$ breaks up into the $\mathbf{1 0}$ and the $\mathbf{5}$ of $\mathrm{Sp}(4, \mathbb{R})$, the former having the right symmetry property to fit in (16), the latter has to drop out. 


\section{The reduction of $\mathrm{SU}(2,2 / N)$ scalar supersingletons to two $\operatorname{OSp}(N / 4, \mathbb{R})$ mirror multiplets}

In this section we explain the mechanism of the decomposition of the $d=4$ scalar supersingletons (massless scalar multiplets of $\mathrm{SU}(2,2 / N)$ ) into a pair of scalar multiplets of the $d=3$ superconformal algebra $\operatorname{OSp}(N / 4, \mathbb{R}) \subset \mathrm{SU}(2,2 / N)$.

We start with the simplest case $N=1$ which illustrates the origin of the shadow scalars in $d=3$. The $d=4$ supersingleton multiplet consists of massless scalars and spinors. It is described by an ultrashort superfield (chiral in the case $N=1$ ). Its $\theta$ expansion contains a term with first-order derivatives of the scalars. Unlike standard trivial dimensional reduction to $d=3$, our procedure consists in keeping the derivative $\partial_{3}$ of the scalars. It plays the rôle of the additional creation operator $P_{3}$ described in Section 2 and is thus responsible for the origin of the shadow scalars.

Next we discuss in detail the case with $N=2$ extended supersymmetry, as well as the generalization to an arbitrary even value of $N$. This time the $d=4$ supersingletons are not chiral, but Grassmann analytic ultrashort superfields in harmonic superspace [15, 16, 17]. Here we see a new feature: The scalars in $d=4$ form an irrep $R_{4}$ of the $d=4 \mathrm{R}$ symmetry group $\mathrm{SU}(N)$ which splits into two inequivalent halves under the $d=3$ group $\mathrm{SO}(N), R_{4}=R_{3}^{+}+R_{3}^{-}$. Accordingly, the $d=4$ supersingleton decomposes into two "mirror" $d=3$ supermultiplets. One of them has scalars of dimension 1 in, e.g., $R_{3}^{+}$as its "ground state" and also contains scalars of dimension 2 in $R_{3}^{-}$. The latter play the rôle of the "shadows" of the ground state scalars of the mirror multiplet.

Another peculiarity of the reduction $\mathrm{SU}(2,2 / N) \rightarrow \operatorname{OSp}(N / 4, \mathbb{R})$ is that the $d=3$ supermultiplets can be viewed as "supercurrents", i.e., as the squares of the $d=3$ scalar supersingletons. This implies that such representations correspond to "massless" fields in $\mathrm{AdS}_{4}$ [6, 9, 18]. For $N \geq 2$ there are two inequivalent species of $d=3$ scalar supersingletons. Each of them gives rise to one of the mirror supercurrent multiplets obtained by decomposing the $d=4$ scalar supersingleton.

\section{1 $\quad N=1$}

The $d=4 N=1$ scalar supersingleton (the massless Wess-Zumino multiplet) is described by a chiral superfield $W(x, \theta, \bar{\theta})$ subject to the massless field equation:

$$
\bar{D}_{\dot{\alpha}} W=D^{\alpha} D_{\alpha} W=0 .
$$

The component expansion of this superfield has the form

$$
W=\phi(x)+\theta^{\alpha} \psi_{\alpha}(x)+i \theta^{\alpha} \sigma_{\alpha \dot{\alpha}}^{\mu} \bar{\theta}^{\dot{\alpha}} \partial_{\mu} \phi(x),
$$

where the complex scalar $\phi(x)$ and the left-handed spinor $\psi_{\alpha}(x)$ fields are massless:

$$
\square \phi=\tilde{\sigma}_{\mu}^{\dot{\alpha} \alpha} \partial^{\mu} \psi_{\alpha}=0 .
$$

Note that the space-time coordinates $x^{\mu}$ in (19) are real. The derivative term originates from the nilpotent shift defining the complex chiral basis $x_{L}^{\mu}=x^{\mu}+i \theta^{\alpha} \sigma_{\alpha \dot{\alpha}}^{\mu} \bar{\theta}^{\dot{\alpha}}$, in which $W$ depends on $\theta$ only. This property is called "chirality", which is a particular case of "Grassmann analyticity" (or " $1 / 2$ BPS shortness"). In fact, the superfield (19) is even "ultrashort", since the term $\theta^{\alpha} \theta_{\alpha}$ is missing. This is a characteristic feature of the supersingleton superfields. 
The reduction to three dimensions is achieved by identifying the two conjugate Grassmann variables

$$
\theta_{\alpha}=\bar{\theta}_{\dot{\alpha}}
$$

and by taking, e.g., the real part of the superfield $W, J=\operatorname{Re} W$. We stress the important difference between the procedure we follow here and the standard trivial dimensional reduction. In the latter case we would also set $\partial_{3} \phi=0$. Then, since the $d=4$ sigma matrices $\sigma_{\alpha \dot{\alpha}}^{\mu}$ split into the $d=3$ ones $\sigma_{(\alpha \beta)}^{m}$ (symmetric) and $\sigma_{\alpha \beta}^{3}=i \epsilon_{\alpha \beta}$, the derivative term in (19) would drop out and we would get the short real $d=3$ superfield $J=A+i \theta^{\alpha} \lambda_{\alpha}$, where $A=\operatorname{Re} \phi, \lambda=\operatorname{Im} \psi$. Such a superfield satisfies the superspace constraint $D^{\alpha} D_{\alpha} J=0$, which is easily shown to be superconformal only if the conformal dimension of the superfield $J$ equals $1 / 2$ and not 1 (the dimension of $W$ ). This is an indication that we have to proceed differently.

The correct dimensional reduction procedure which preserves conformal invariance, consists in keeping $\partial_{3} \phi$. According to the discussion in Section 2, this corresponds to considering $\partial_{3}$ as the component of the raising operator $P_{3}$ which is responsible for creating a new scalar state. In terms of fields this means defining a new $d=3$ field $B=-\operatorname{Im} \partial_{3} \phi$. Thus, we obtain a generic long real|2 $d=3$ superfield:

$$
\begin{gathered}
J=A(x)+i \theta^{\alpha} \lambda_{\alpha}(x)+i \theta^{\alpha} \theta_{\alpha} B(x), \\
A=\operatorname{Re} \phi, \quad \lambda_{\alpha}=\operatorname{Im} \psi_{\alpha}, \quad B=-\operatorname{Im} \partial_{3} \phi .
\end{gathered}
$$

In the process the first scalar $A$ keeps the original conformal dimension 1 of $\phi$, but the new scalar $B$ acquires the "shadow" dimension $3-1=2$.

Instead of the real part of $W$, we could have taken its imaginary part, thus obtaining an alternative ("mirror") $d=3$ supermultiplet:

$$
\begin{gathered}
J^{\prime}=A^{\prime}(x)+i \theta^{\alpha} \lambda_{\alpha}^{\prime}(x)+i \theta^{\alpha} \theta_{\alpha} B^{\prime}(x), \\
A^{\prime}=\operatorname{Im} \phi, \quad \lambda_{\alpha}^{\prime}=-\operatorname{Re} \psi_{\alpha}, \quad B^{\prime}=\operatorname{Re} \partial_{3} \phi .
\end{gathered}
$$

The only difference between $J$ and $J^{\prime}$ has to do with the fact that real part of $\phi$ is a scalar while its imaginary part is a pseudoscalar. Later on we shall see that the difference between the mirror $d=3$ multiplets becomes more significant for $N>1$.

Finally, $J$ can be viewed as a "supercurrent" obtained from $d=3$ supersingletons $\Phi(x, \theta)$ defined by the massless superfield equation

$$
D^{\alpha} D_{\alpha} \Phi=0 \Rightarrow \Phi=\omega(x)+\theta^{\alpha} \chi_{\alpha}, \quad \square \omega=\partial^{\alpha \beta} \chi_{\beta}=0 .
$$

Note that this time the dimensions of the $d=3$ fields are canonical, $\operatorname{dim} \omega=1 / 2, \operatorname{dim} \chi=1$, so that equation (24) can be superconformal. Then we can write the "supercurrent" $J=(\Phi)^{2}$ which has the same content as (22). The term "supercurrent" will become more clear when we move to $N>2$, where we will find conserved currents among the components of $J$.

\footnotetext{
${ }^{2}$ This is the reason why we had to expand the chiral superfield $W(\sqrt{19})$ in the real basis in superspace, and not in the complex chiral basis.
} 


\section{$4.2 \quad N=2$}

The $d=4 N=2$ scalar supersingleton is the hypermultiplet. Its adequate description as a Grassmann analytic superfield is given in harmonic superspace [15]. To this end one introduces harmonic variables $u_{i}^{I}$ (and their complex conjugates $u_{I}^{i}$ ) parametrizing the coset $\mathrm{SU}(2) / \mathrm{U}(1)$. They form a matrix of the R symmetry group $\mathrm{SU}(2)$ whose index $i$ transforms under the fundamental representation of $\mathrm{SU}(2)$, whereas the index $I$ is a collection of $\mathrm{U}(1)$ charges. With the help of the harmonics we can covariantly project any $\mathrm{SU}(2)$ representation with respect to the subgroup U(1). For instance, the Grassmann variables $\bar{\theta}_{\dot{\alpha}}^{i}$ and $\theta_{i \alpha}$ in the fundamental irrep of $\mathrm{SU}(2)$ can be projected onto the highest-weight states (HWS) $\bar{\theta}_{\dot{\alpha}}^{1}=\bar{\theta}_{\dot{\alpha}}^{i} u_{i}^{1}, \theta_{2 \alpha}=\theta_{i \alpha} u_{2}^{i}$, etc. Note the existence of a particular conjugation (real structure) which combines ordinary complex conjugation with the antipodal map on the sphere $S^{2} \sim \mathrm{SU}(2) / \mathrm{U}(1)$ :

$$
\widetilde{\theta}_{2 \alpha}=\bar{\theta}_{\dot{\alpha}}^{1}
$$

The on-shell hypermultiplet can now be described by a Grassmann analytic superfield:

$$
W^{1}\left(x_{A}, \bar{\theta}^{1}, \theta_{2}, u\right)=\phi^{i}\left(x_{A}\right) u_{i}^{1}+\theta_{2}^{\alpha} \psi_{\alpha}\left(x_{A}\right)+\bar{\theta}^{1} \dot{\alpha}_{\dot{\alpha}}\left(x_{A}\right)+i \theta_{2} \sigma^{\mu} \bar{\theta}^{1} \partial_{\mu} \phi^{i}\left(x_{A}\right) u_{i}^{2},
$$

where the component fields are massless,

$$
\square \phi^{i}=\tilde{\partial}^{\dot{\alpha} \alpha} \psi_{\alpha}=\partial_{\alpha \dot{\alpha}} \bar{\kappa}^{\dot{\alpha}}=0 .
$$

The space-time variables $x_{A}^{\mu}$ in $(26)$ are obtained from the real ones $x^{\mu}$ by a nilpotent shift, $x_{A}^{\mu}=x^{\mu}+i \theta_{2} \sigma^{\mu} \bar{\theta}^{2}-i \theta_{1} \sigma^{\mu} \bar{\theta}^{1}$. In this basis the Grassmann analytic superfield manifestly depends only on half of the odd variables. In this respect the Grassmann analytic superfields resemble the chiral ones, although the half of $\theta$ 's they depend on is chosen with regard to the $\mathrm{R}$ symmetry instead of the Lorentz group. We can also say that this is another type of $1 / 2$ BPS short superfield.

In addition to Grassmann analyticity, $W^{1}$ is subject to the constraint of $\mathrm{SU}(2)$ irreducibility (harmonic analyticity), which makes the harmonic dependence in $(\sqrt[26]{)})$ linear and puts the physical fields on shell. As in the case $N=1$, the supersingleton (26) is an "ultrashort" superfield, in the sense that a number of components in its $\theta$ expansion are missing.

One final remark concerns the derivative term $i \theta_{2} \sigma^{\mu} \bar{\theta}^{1} \partial_{\mu} \phi^{i}\left(x_{A}\right) u_{i}^{2}$. It is not the analog of the coordinate shift term in the $N=1$ chiral superfield (19), but is due to the ultrashortness of the supersingleton. This term will play an important rôle in the dimensional reduction.

Now we proceed to the reduction to three dimensions. There the $N=2 \mathrm{R}$ symmetry group $\mathrm{SU}(2)$ becomes $\mathrm{SO}(2)$ and the Grassmann variables belong to the vector representation, $\theta^{ \pm \pm \alpha}$. Thus, to reduce the $d=4$ superfield (26) we have to identify the two conjugate (in the sense of eq. (25)) $\theta$ 's,

$$
\theta_{2}^{\alpha}=\bar{\theta}^{1 \dot{\alpha}} \equiv \theta^{++\alpha}
$$

Further, we define the scalar field $A^{++} \equiv \phi^{i} u_{i}^{1}$ and the spinor field $\lambda^{\alpha} \equiv 1 / 2\left(\psi^{\alpha}+\bar{\kappa}^{\dot{\alpha}}\right)$. Finally, much like in the case $N=1$, from the derivative term in (26) only the component $B^{--} \equiv$ $-\partial_{3} \phi^{i} u_{i}^{2}$ survives, giving rise to a new scalar field of shadow dimension 2 . The result is

$$
J^{++}=A^{++}+\theta^{++\alpha} \lambda_{\alpha}+\theta^{++\alpha} \theta_{\alpha}^{++} B^{--} .
$$

\footnotetext{
${ }^{3}$ We use spinor units of charge, so the charge of a vector is \pm 2 .
} 
Unlike the case $N=1$, this time we obtain a $1 / 2$ BPS short (or Grassmann analytic) superfield depending only on half of the odd variables.f However, this short superfield is off shell, unlike its $d=4$ counterpart (26).

A characteristic feature of the $d=3$ supermultiplet (29), common for all even values of $N$, is that its "ground state" $A^{++}$corresponds to half of the $d=4$ scalars $\phi^{i}$. It is obtained by splitting the $\mathrm{R}$ symmetry $n$-fold antisymmetric irrep of $\mathrm{SU}(2 n)$ into two inequivalent irreps (self-dual and anti-self-dual) of $\mathrm{SO}(2 n)$. The other half reappears at the level of $(\theta)^{2}$, but this time with shadow dimension 2.

Choosing the other half of the scalars as the "ground state" of the supermultiplet, we find an alternative ("mirror") reduction of the $d=4$ supersingleton. It is obtained from the complex conjugate superfield $\bar{W}_{2}\left(\theta_{1}, \bar{\theta}^{2}\right)=\bar{\phi}_{i} u_{2}^{i}+\ldots$ by the identifications $\theta_{1}^{\alpha}=\bar{\theta}^{2 \dot{\alpha}} \equiv \theta^{--\alpha}, A^{--}=$ $\bar{\phi}_{i} u_{2}^{i}, \rho^{\alpha} \equiv 1 / 2\left(\psi^{\alpha}-\bar{\kappa}^{\dot{\alpha}}\right)$ and $B^{++} \equiv-\partial_{3} \bar{\phi}_{i} u_{2}^{i}$ :

$$
J^{--}=A^{--}+\theta^{--\alpha} \rho_{\alpha}+\theta^{--\alpha} \theta_{\alpha}^{--} B^{++} .
$$

Notice the important fact that the "shadow" scalar $B^{++}$in (30) carries the same $\mathrm{R}$ symmetry quantum numbers as the "ground state" scalar $A^{++}$in (29). In other words, the pair of $d=3$ scalars which gives rise to a singleton scalar representation of $\mathrm{SO}(4,2)$ (recall Section 2), is composed of states belonging to the two "mirror" $d=3$ supermultiplets (29) and (30). We conclude that this pair of $d=3$ supermultiplets is equivalent to the $d=4$ supersingleton multiplet (26). As we show below, the same phenomenon takes place for all even values of $N$.

Finally, we remark that the $d=3 N=2$ supermultiplet we have obtained is a "supercurrent", i.e., it can be viewed as the square of a $d=3$ supersingleton. The latter is described by an ultrashort analytic superfield,

$$
\Phi^{+}=\omega^{+}+\theta^{++\alpha} \chi_{\alpha}^{-}, \quad \square \omega^{+}=\partial^{\alpha \beta} \chi_{\beta}^{-}=0 \Rightarrow J^{++}=\left(\Phi^{+}\right)^{2},
$$

or, alternatively, by $\Phi^{-}=\omega^{-}+\theta^{--\alpha} \chi_{\alpha}^{+}, J^{--}=\left(\Phi^{-}\right)^{2}$.

\subsection{Arbitrary even $N$}

The above discussion is easily generalized to the case of an arbitrary even value $N=2 n$. Two examples of $d=4$ supersingletons of physical interest are the on-shell multiplets of $N=4$ super Yang-Mills and of $N=8$ supergravity [19].

\subsubsection{Scalar supersingletons in $d=4$}

The $d=4 N=2 n$ scalar supersingleton is described by an ultrashort analytic superfield. It depends on half of the Grassmann variables:

$$
\bar{\theta}^{1,2, \cdots, n \dot{\alpha}}=\bar{\theta}^{i \dot{\alpha}} u_{i}^{1,2, \cdots, n}, \quad \theta_{n+1, \cdots, 2 n}^{\alpha}=\theta_{i}^{\alpha} u_{n+1, \cdots, 2 n}^{i},
$$

obtained with the help of the harmonic variables $u_{i}^{I}$ (and their conjugates $u_{I}^{i}$ ) on the coset $\mathrm{SU}(2 n) /[\mathrm{U}(1)]^{2 n-1}[20]$. They form an $\mathrm{SU}(2 n)$ matrix and are used to project the fundamental irrep of $\mathrm{SU}(2 n)$ (index $i$ ) onto $[\mathrm{U}(1)]^{2 n-1}$ (index $I=1,2, \ldots, 2 n$ ).

\footnotetext{
${ }^{4}$ The space-time variables in $(29)$ include the nilpotent shift $x_{A}^{m}=x^{m}+i \theta^{++} \sigma^{m} \theta^{--}$which originates from the corresponding shift in $d=4$.
} 
Below we list some of the terms in the $\theta$ expansion of the supersingleton:

$$
\begin{aligned}
W^{12 \cdots n}\left(\bar{\theta}^{1,2, \cdots, n}, \theta_{n+1, \cdots, 2 n}\right) & =\phi^{\left[i_{1} \cdots i_{n}\right]} u_{i_{1}}^{1} \cdots u_{i_{n}}^{n} \\
& +\bar{\theta}^{n \dot{\alpha}} \bar{\psi}_{\dot{\alpha}}^{\left[i_{1} \cdots i_{n-1}\right]} u_{i_{1}}^{1} \cdots u_{i_{n-1}}^{n-1}+\text { perm. } \\
& +\theta_{2 n}^{\alpha} \kappa_{\left[i_{1} \cdots i_{n-1}\right] \alpha} u_{2 n-1}^{i_{1}} \cdots u_{n+1}^{i_{n-1}}+\text { perm. } \\
& +\cdots \\
& +\bar{\theta}_{\dot{\alpha}_{1}}^{1} \cdots \bar{\theta}_{\dot{\alpha}_{n}}^{n} F^{\left(\dot{\alpha}_{1} \cdots \dot{\alpha}_{n}\right)}+\theta_{2 n}^{\alpha_{1}} \cdots \theta_{n+1}^{\alpha_{n}} G_{\left(\alpha_{1} \cdots \alpha_{n}\right)} \\
& +i \theta_{2 n} \sigma^{\mu} \bar{\theta}^{n} \partial_{\mu} \phi^{\left[i_{1} \cdots i_{n}\right]} u_{i_{1}}^{1} \cdots u_{i_{n-1}}^{n-1} u_{i_{n}}^{2 n}+\text { perm. } \\
& + \text { other derivative terms }
\end{aligned}
$$

These include: the scalars in the $n$-fold antisymmetric irrep $\left[0 \cdots 01_{n} 0 \cdots 0_{2 n-1}\right]$ of $\mathrm{SU}(2 n)$; the spin $1 / 2$ fields in the $n-1$-fold antisymmetric irrep $\left[0 \cdots 01_{n-1} 0 \cdots 0_{2 n-1}\right]$; the $\mathrm{SU}(2 n)$ singlets $F$ and $G$ with the top Lorentz spins $(0, n)$ and $(n, 0)$; the derivative terms relevant to the dimensional reduction. All of these fields are massless,

$$
\square \phi=\partial_{\alpha \dot{\alpha}} \bar{\psi}^{\dot{\alpha}}=\tilde{\partial}^{\dot{\alpha} \alpha} \kappa_{\alpha}=\ldots=\partial_{\alpha \dot{\alpha}_{1}} F^{\left(\dot{\alpha}_{1} \cdots \dot{\alpha}_{n}\right)}=\tilde{\partial}^{\dot{\alpha} \alpha_{1}} G_{\left(\alpha_{1} \cdots \alpha_{n}\right)}=0 .
$$

The harmonic projections of the fields displayed in (33) correspond to the HWS of each $\mathrm{SU}(2 n)$ irrep, the rest are obtained by permutation of the indices $1,2, \ldots, 2 n$. The conjugation rules compatible with Grassmann analyticity are as follows:

$$
\widetilde{\theta}_{n+1}=\bar{\theta}^{1}, \ldots, \widetilde{\theta}_{2 n}=\bar{\theta}^{n} .
$$

Using these rules, we can impose a reality condition on the multiplet (33) if $N=2 n=4 k$.

To be more explicit, let us give the particular example of the $N=4$ SYM multiplet in detail:

$$
\begin{aligned}
W_{N=4 \mathrm{SYM}}^{12}\left(\bar{\theta}^{1,2}, \theta_{3,4}\right) & =\phi^{[i j]} u_{i}^{1} u_{j}^{2} \\
& +\left(\bar{\theta}^{2 \dot{\alpha}} \bar{\psi}_{\dot{\alpha}}^{i} u_{i}^{1}-1 \leftrightarrow 2\right)+\left(\theta_{4}^{\alpha} \psi_{\alpha i} u_{3}^{i}-3 \leftrightarrow 4\right) \\
& +\bar{\theta}^{1 \dot{\alpha}} \bar{\theta}^{2 \dot{\beta}} F_{(\dot{\alpha} \dot{\beta})}^{-}+\theta_{4}^{\alpha} \theta_{3}^{\beta} F_{(\alpha \beta)}^{+} \\
& +\left(\left(i \theta_{4} \sigma^{\mu} \bar{\theta}^{2} \partial_{\mu} \phi^{[i j]} u_{i}^{1} u_{j}^{4}+3 \leftrightarrow 4\right)-1 \leftrightarrow 2\right)+\text { other derivative terms } .
\end{aligned}
$$

It consists of six scalars $\phi^{i j}=1 / 2 \epsilon^{i j k l} \bar{\phi}_{k l}$ in the real irrep [010] of SU(4), four spinors $\bar{\psi}_{\dot{\alpha}}^{i}$ in the fundamental irrep [100] (and their conjugates $\psi_{\alpha i}$ in [001]) and the self-dual $F_{(\alpha \beta)}^{+}$and anti-self-dual $F_{(\dot{\alpha} \dot{\beta})}^{-}$parts of the YM field strength (SU(4) singlets).

In eqs. (33) and(36) we have displayed only one of the numerous derivative terms. The remainder contains either derivatives of the spinors or higher-order derivatives of the scalars. They are irrelevant for the dimensional reduction because of the field equations (34). Indeed, a term like $\partial_{\mu} \psi_{\alpha}$ will only generate derivative terms (descendants) in $d=3$, since $\partial_{3} \psi_{\alpha}=\tilde{\sigma}_{3}^{\alpha \dot{\alpha}} \sigma_{\dot{\alpha} \beta}^{m} \partial_{m} \psi^{\beta}$. Similarly, since $\partial_{3}^{2} \phi=\partial_{m}^{2} \phi$, derivative terms like $\partial_{\mu} \partial_{\nu} \phi$ either give rise to descendants of the $d=3$ field $A \equiv \phi$ or of the new $d=3$ field $B \equiv \partial_{3} \phi$ (see below).

\subsubsection{Reduction to $d=3$}

By analogy with the case $N=2$, after the reduction to $d=3$ we expect to find an $\mathrm{SO}(2 \mathrm{n})$ covariant analytic superfield. In order to define it we have to introduce harmonic variables on 
the coset $\mathrm{SO}(2 n) /[\mathrm{U}(1)]^{n}$. 5 A characteristic feature of the orthogonal groups $\mathrm{SO}(2 n)$ (more precisely, of their covering groups $\operatorname{Spin}(2 n)$ ) is the existence of two inequivalent spinor representations (left- and right-handed), denoted by undotted and dotted indices $a, \dot{a}=1, \ldots, 2^{n-1}$. Correspondingly, we introduce two sets of spinor harmonics,

$$
u_{a}^{ \pm_{1} \cdots \pm_{n-2}[ \pm]}, \quad w_{\dot{a}}^{ \pm_{1} \cdots \pm_{n-2}\{ \pm\}} .
$$

Their $\mathrm{U}(1)$ charges denoted by $\pm_{p}, p=1, \ldots, n-2$ correspond to the first $n-2 \mathrm{U}(1)$ factors from the coset $\mathrm{SO}(2 n) /[\mathrm{U}(1)]^{n}$. The last two charges, denoted by $[ \pm]$ and $\{ \pm\}$, are used to distinguish the two spinor representations whose HWS correspond to the projections

$$
\left[0 \cdots 010_{n}\right]: u_{a}^{+{ }^{\cdots} \cdots+_{n-2}[+]} ; \quad\left[0 \cdots 01_{n}\right]: w_{\dot{a}}^{+_{1} \cdots+_{n-2}\{+\}} .
$$

Further, in $d=4$ the Grassmann variables $\theta_{i}$ and $\bar{\theta}^{i}$ belong to the fundamental irrep of $\mathrm{SU}(2 n)$, but after reducing to $d=3$ and taking the real part, we find them in the vector irrep of $\mathrm{SO}(2 n)$. The corresponding vector harmonics are composite variables made out of the left- and right-handed spinor harmonics,

$$
v_{r}^{R}=u_{a}^{ \pm_{1} \cdots \pm_{n-2}[ \pm]} \gamma_{r}^{a \dot{a}} w_{\dot{a}}^{ \pm_{1} \cdots \pm_{n-2}\{ \pm\}},
$$

where $\gamma_{r}, r=1, \ldots, 2 n$ are the gamma matrices of $\mathrm{SO}(2 n)$. The projection (charge) $R$ takes one of the following $2 n$ values: $\pm_{ \pm_{1}}$ or $\pm_{ \pm_{2}}, \ldots$, or $\pm \pm_{n-2}$, or $[ \pm]\{ \pm\}$. The HWS of the vector irrep $\left[10 \cdots 0_{n}\right]$ corresponds to the projection $v_{r}^{++1}($ if $n>2)$ or to $v^{[+]\{+\}}$(if $\left.n=2\right)$.

Using the composite vector harmonics, we can choose a subset of the odd variables suitable for defining a $d=3$ analytic superspace. One such subset is obtained by identifying the left- and right-handed $d=4$ analytic $\theta$ 's as follows:

$$
\theta_{n+1}=\bar{\theta}^{1} \equiv \theta^{++1}, \ldots, \theta_{2 n-2}=\bar{\theta}^{n-2} \equiv \theta^{++n-2}, \theta_{2 n-1}=\bar{\theta}^{n-1} \equiv \theta^{[+]\{+\}}, \theta_{2 n}=\bar{\theta}^{n} \equiv \theta^{[+]\{-\}} .
$$

These new odd variables are real, as follows from the conjugation rules (35). Another analytic subset is obtained by swapping $\theta^{[+]\{ \pm\}} \leftrightarrow \theta^{[ \pm]\{+\}}$.

The next step in the reduction is to split the scalars in eq. (33) into irreps of $\mathrm{SO}(2 n)$. In fact, all other fields in (33) belong to $\mathrm{SU}(2 n)$ irreps of the type $\left[0 \cdots 01_{p} 0 \cdots 0_{2 n-1}\right], 1 \leq p \leq n-1$, which remain irreducible when restricted to $\mathrm{SO}(2 n)$ (more precisely, to $\operatorname{Spin}(2 n)$ ). The only exception are the scalars in the $\left[0 \cdots 01_{n} 0 \cdots 0_{2 n-1}\right]$ of $\mathrm{SU}(2 n)$ which split into the left- and right-handed irreps $\left[0 \cdots 020_{n}\right]$ and $\left[0 \cdots 02_{n}\right]$ of $\mathrm{SO}(2 n)$ (corresponding to self-dual and anti-self-dual $n$-fold antisymmetric tensors). We can take, e.g., the self-dual half $\left[0 \cdots 020_{n}\right]$ (i.e., the HWS charges $\left.++_{1} \cdots++_{n-2}[++]\right)$ and define the $d=3$ "ground state" scalar fields

$$
\phi^{\left[i_{1} \cdots i_{n}\right]} u_{i_{1}}^{1} \cdots u_{i_{n}}^{n} \rightarrow A^{++1_{1} \cdots++{ }_{n-2}[++]}=A^{\left[r_{1} \cdots r_{n}\right]} v_{r_{1}}^{++1} \cdots v_{r_{n-2}}^{++n-2} v_{r_{n-1}}^{[+]\{+\}} v_{r_{n}}^{[+]\{-\}} .
$$

Here $A^{\left[r_{1} \cdots r_{n}\right]}$ is an $n$-fold antisymmetric self-dual tensor of $\mathrm{SO}(2 n)$. If $n=2 k$ it can be made real by applying the appropriate harmonic conjugation.

In a similar fashion, the remaining terms in the $d=4$ expansion (33) give rise to $d=3$ fields. For instance, the spin $1 / 2$ terms become

$$
\bar{\theta}^{n \dot{\alpha}} \bar{\psi}_{\dot{\alpha}}^{12 \cdots n-1}+\theta_{2 n}^{\alpha} \kappa_{2 n-1 \cdots n+1 \alpha} \rightarrow \theta^{[+]\{-\} \alpha} \lambda_{\alpha}^{+++1 \cdots++{ }_{n-2}[+]\{+\}},
$$

\footnotetext{
${ }^{5}$ We did not need harmonics in the case $N=2$ since the group $\mathrm{SO}(2)$ is too small to admit a harmonic coset.
} 
where $\lambda_{\alpha}^{++{ }_{1} \cdots++_{n-2}[+]\{+\}}=\lambda_{\alpha\left[r_{1} \cdots r_{n-1}\right]} v_{r_{1}}^{++1} \cdots v_{r_{n-2}}^{++{ }_{n-2}} v_{r_{n-1}}^{[+]\{+\}}$is a spinor filed corresponding to

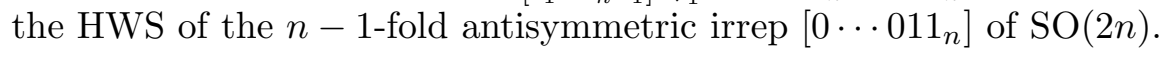

This process goes on until we reach the top spin fields:

$$
\bar{\theta}_{\dot{\alpha}_{1}}^{1} \cdots \bar{\theta}_{\dot{\alpha}_{n}}^{n} F^{\left(\dot{\alpha}_{1} \cdots \dot{\alpha}_{n}\right)}+\theta_{2 n}^{\alpha_{1}} \cdots \theta_{n+1}^{\alpha_{n}} G_{\left(\alpha_{1} \cdots \alpha_{n}\right)} \rightarrow \theta_{\alpha_{1}}^{++1} \cdots \theta_{\alpha_{n-2}}^{++{ }_{n}-2} \theta_{\alpha_{n-1}}^{[+]\{+\}} \theta_{\alpha_{n}}^{[+]\{-\}} j^{\left(\alpha_{1} \cdots \alpha_{n}\right)} .
$$

Note that the spin $n$ filed $j^{\left(\alpha_{1} \cdots \alpha_{n}\right)}$ (as well as all other fields with spin greater than $1 / 2$ ) is a conserved spin-tensor:

$$
\sigma_{\alpha_{1} \alpha_{2}}^{m} \partial_{m} j^{\left(\alpha_{1} \cdots \alpha_{n}\right)}=0 .
$$

The reason for this will become clear in subsection 4.4.

The derivative term shown in (33) deserves particular attention, because it gives rise to the new scalars of shadow dimension 2:

$$
i \theta_{2 n} \sigma^{3} \bar{\theta}^{n} \partial_{3} \phi^{1 \cdots n-1 ; 2 n} \rightarrow \theta^{[+]\{-\} \alpha} \theta_{\alpha}^{[+]\{-\}} B^{++1_{1} \cdots++{ }_{n-2}\{++\}},
$$

where $B^{+++_{1} \cdots++{ }_{n-2}\{++\}}=B_{\left[r_{1} \cdots r_{n}\right]} v_{r_{1}}^{++1} \cdots v_{r_{n-2}}^{++n-2} v_{r_{n-1}}^{[+]\{+\}} v_{r_{n}}^{[-]\{+\}}$is a scalar filed corresponding to the HWS of the $n$-fold antisymmetric anti-self-dual irrep $\left[0 \cdots 02_{n}\right]$.

As in the case $N=2$, the "ground state" scalars $A$ and the "shadow" scalars $B$ belong to the two complementary $\mathrm{SO}(2 n)$ irreps $\left[0 \cdots 020_{n}\right]$ and $\left[0 \cdots 02_{n}\right]$ which constitute the $\mathrm{SU}(2 n)$ irrep $\left[0 \cdots 01_{n} 0 \cdots 0_{2 n-1}\right]$. This means that the shadows of the $A$ fields (41) are not the $B$ fields (45) from the analytic superfield $J^{\left[0 \cdots 020_{n}\right]}\left(\theta^{++1}, \ldots, \theta^{++_{n-2}}, \theta^{[+]\{ \pm\}}\right)$that we have constructed. They can be found in the "mirror" superfield $J^{\left[0 \cdots 02_{n}\right]}\left(\theta^{++1}, \ldots, \theta^{++{ }_{n-2}}, \theta^{[ \pm]\{+\}}\right)$obtained by swapping the irreps of the "ground state" and of the shadow scalars (in practice, this means swapping the charges $[ \pm]$ and $\{ \pm\}$ ). We conclude that the $d=4$ supersingleton decomposes into two "mirror" $d=3$ multiplets, each of them containing the shadow scalars for the other.

Concluding this subsection, we give as an example the complete expansion of the $d=3 N=4$ analytic superfield obtained from the $d=4 N=4 \mathrm{SYM}$ supersingleton:

$$
\begin{aligned}
J_{N=4}^{[++]} \mathrm{SYM} & =A^{[++]} \\
& +\theta^{[+]\{-\} \alpha} \lambda_{\alpha}^{[+]\{+\}}-\theta^{[+]\{+\} \alpha} \lambda_{\alpha}^{[+]\{-\}} \\
& +\theta^{[+]\{-\} \alpha} \theta_{\alpha}^{[+]\{-\}} B^{\{++\}}-\theta^{[+]\{-\} \alpha} \theta_{\alpha}^{[+]\{+\}} B^{\{-+\}}+\theta^{[+]\{+\} \alpha} \theta_{\alpha}^{[+]\{+\}} B^{\{--\}} \\
& +\theta^{[+]\{+\} \alpha} \sigma_{\alpha \beta}^{m} \theta^{[+]\{-\} \beta} j_{m} \\
& + \text { derivative terms }
\end{aligned}
$$

where the vector $j_{m}$ is conserved, $\partial^{m} j_{m}=0$.

\subsection{Supercurrent multiplets}

In the cases $N=1,2$ we have seen that the two $d=3$ supermultiplets obtained by reducing the $d=4$ scalar supersingleton can be regarded as "supercurrents", i.e., as the squares of the $d=3$ supersingletons. The same is true for any $N=2 n$. For instance, in the case $N=4$ there are two types of such $d=3$ supersingletons, differing by the $\mathrm{SO}(4)$ assignments of the scalars and the spinors. The supersingleton corresponding to the supermultiplet (46) is described by the following ultrashort analytic superfield:

$$
\Phi^{[+]}=\omega_{a} u_{a}^{[+]}+\theta^{[+]\{-\} \alpha} \chi_{\alpha \dot{a}} w_{\dot{a}}^{\{+\}}-\theta^{[+]\{+\} \alpha} \chi_{\alpha \dot{a}} w_{\dot{a}}^{\{-\}}+\theta^{[+]\{+\}} \sigma^{m} \theta^{[+]\{-\}} i \partial_{m} \omega_{a} u_{a}^{[-]},
$$


where $\square \omega_{a}=\partial^{\alpha \beta} \chi_{\beta}=0$. Then the "supercurrent" (46) is simply the square of the supersingleton $(47), J^{[++]}=\left(\Phi^{[+]}\right)^{2}$. The alternative supersingleton $\Phi^{\{+\}}$has the mirror $\mathrm{SO}(4)$ assignments and gives rise to the mirror supercurrent $J^{\{++\}}=\left(\Phi^{\{+\}}\right)^{2}$. This realisation of the supermultiplet (46) explains why the vector $j_{m}$ is conserved: It is simply the current $j_{m}=i \omega^{a} \partial_{m} \omega_{a}+\chi^{\dot{a}} \sigma_{m} \chi_{\dot{a}}$. The generalization to any $N=2 n$ is straightforward. The supermultiplet $J^{\left[0 \cdots 020_{n}\right]}$ can be viewed as a "supercurrent", i.e., as the square of the corresponding $d=3$ supersingleton

$$
\begin{gathered}
\Phi^{+1 \cdots+{ }_{n-2}[+]}=\omega_{a} u_{a}^{+1 \cdots+{ }_{n-2}[+]}+\left(\theta^{[+]\{-\} \alpha} \chi_{\alpha \dot{a}} w_{\dot{a}}^{+1 \cdots+{ }_{n-2}\{+\}}+\text { perm. }\right)+\text { der. terms } \\
\Rightarrow \quad J^{\left[0 \cdots 020_{n}\right]} \equiv J^{++_{1} \cdots++_{n-2}[++]}=\left(\Phi^{+{ }_{1} \cdots+{ }_{n-2}[+]}\right)^{2}
\end{gathered}
$$

(and similarly for the mirror multiplet).

\section{Reducing the $\mathrm{F}(4)$ supersingleton to $\mathrm{SU}(2,2 / 1)$ multiplets}

As explained in Section 2, the unique $d=5$ superconformal algebra $\mathrm{F}(4)$ can be reduced to the $d=4 N=1$ superalgebra $\mathrm{SU}(2,2 / 1)$. In this case we find that the $d=5$ scalar supersingleton ("hypermultiplet") is reduced to a pair of $d=4$ scalar supermultiplets with "anomalous" dimension $3 / 2$. Here are the details.

The $d=5$ pseudo-Majorana spinors $\theta_{\alpha}^{i}=\Omega_{\alpha \beta} \epsilon^{i j}\left(\theta_{\beta}^{j}\right)^{*}$ carry two kinds of indices: $\alpha=1, \ldots, 4$ is a spinor index of $\mathrm{USp}(2,2) \sim \mathrm{SO}(4,1)$ and $i=1,2$ is a doublet index of $\mathrm{SU}(2)$. The scalar supersingleton is very similar to the $d=4 N=2$ hypermultiplet described in Section 4.2. Introducing the same $\mathrm{SU}(2) / \mathrm{U}(1)$ harmonic variables and projecting $\theta_{\alpha}^{1,2}=\theta_{\alpha}^{i} u_{i}^{1,2}$, we can write down the ultrashort analytic superfield

$$
W^{1}\left(x_{A}, \theta^{1}, u\right)=\phi^{i}\left(x_{A}\right) u_{i}^{1}+\theta_{\alpha}^{1} \psi^{\alpha}\left(x_{A}\right)+i \theta^{1} \gamma^{\mu} \theta^{1} \partial_{\mu} \phi^{i}\left(x_{A}\right) u_{i}^{2},
$$

where the component fields are massless,

$$
\square \phi^{i}=\gamma_{\alpha \beta}^{\mu} \partial_{\mu} \psi^{\alpha}=0 \text {. }
$$

Correspondingly, they have the canonical dimensions

$$
\ell_{\phi}=3 / 2, \quad \ell_{\psi}=2 .
$$

The space-time variables $x_{A}^{\mu}$ in (49) are obtained from the real ones $x^{\mu}$ by a nilpotent shift, $x_{A}^{\mu}=x^{\mu}+i \theta^{1} \gamma^{\mu} \theta^{2}$. The $d=5$ gamma matrices have the properties $\gamma_{\alpha \beta}^{\mu}=-\gamma_{\beta \alpha}^{\mu}, \Omega^{\alpha \beta} \gamma_{\alpha \beta}^{\mu}=0$.

The reduction to $d=4$ is obtained by splitting the four-component $\operatorname{USp}(2,2)$ spinor $\theta_{\alpha}^{1}$ into leftand right-handed spinors of $\operatorname{SL}(2, \mathbb{C}), \theta_{\alpha}^{1} \rightarrow \theta_{\alpha}^{+}, \bar{\theta}_{\dot{\alpha}}^{+}$, both carrying charge +1 under the $d=4$ $\mathrm{R}$ symmetry group $\mathrm{U}(1)$. 5 In order to obtain an irreducible $d=4$ supermultiplet, we keep only, e.g., the left-handed half. Further, the $d=5$ fields in (49) become $d=4$ fields $\phi^{i} u_{i}^{1} \rightarrow A^{+}$, $\psi^{\alpha} \rightarrow \lambda^{\alpha}$ of non-canonical dimensions $3 / 2$ and 2 , respectively. The derivative term in (49), when restricted to left-handed $\theta$ 's only, gives rise to the scalar $B^{-} \equiv \partial_{4} \phi^{i} u_{i}^{2}$ of shadow dimension

\footnotetext{
${ }^{6}$ Note that in Section 4.2 the odd variables were vectors of $\mathrm{SO}(2)$ and carried charges \pm 2 , while here they are treated as spinors of $\mathrm{SO}(2)$ with charges \pm 1 .
} 
$4-3 / 2=5 / 2$. Finally, the analytic basis variables $x_{A}^{\mu}$ in (49) become the left-handed chiral basis variables $x_{L}^{m}=x^{m}+i \theta^{+} \sigma^{m} \bar{\theta}^{-}$. The end result is the $d=4$ chiral superfield

$$
J^{+}\left(x_{L}\right)=A^{+}\left(x_{L}\right)+\theta^{+\alpha} \psi_{\alpha}\left(x_{L}\right)+\theta^{+\alpha} \theta_{\alpha}^{+} B^{-}\left(x_{L}\right)
$$

with "anomalous" dimension $3 / 2$.

Alternatively, starting with the complex conjugate supersingleton and retaining the right-handed halves of the odd variables, we can obtain the mirror $d=4$ chiral multiplet

$$
J^{-}\left(x_{R}\right)=A^{-}\left(x_{R}\right)+\bar{\theta}_{\dot{\alpha}}^{-} \bar{\rho} \dot{\alpha}\left(x_{R}\right)+\bar{\theta}_{\dot{\alpha}}^{-} \bar{\theta}^{-\dot{\alpha}} B^{+}\left(x_{R}\right) .
$$

As before, the two pairs of shadow $d=4$ scalars $A^{ \pm}, B^{ \pm}$needed to form the two $d=5$ singletons are distributed over the two mirror supermultiplets.

The case $d=5 \rightarrow d=4$ differs from $d=4 \rightarrow d=3$ in that the supermultiplets $J^{ \pm}$are not "supercurrents", i.e., they are not obtained as bilinears in the $d=4$ supersingletons (otherwise they would have dimension 2 instead of $3 / 2$ ).

\section{Acknowledgements}

The work of S.F. has been supported in part by the European Commission RTN network HPRNCT-2000-00131 (Laboratori Nazionali di Frascati, INFN) and by the D.O.E. grant DE-FG0391ER40662, Task C. S.F. is grateful to M. Porrati for useful discussions. E.S. acknowledges discussions with G. Arutyunov and A. Petkou.

\section{References}

[1] C. Fronsdal, "Elementary Particles In A Curved Space. 4. Massless Particles," Phys. Rev. D 12 (1975) 3819.

[2] S. Ferrara, R. Gatto, A. Grillo and G. Parisi, "The shadow operator formalism for conformal algebra", Lett. Nuovo Cimento 4 (1972) 115.

[3] O. DeWolfe, D. Z. Freedman and H. Ooguri, "Holography and defect conformal field theories," arXiv:hep-th/0111135.

[4] M. Porrati, "Higgs phenomenon for 4-D gravity in anti de Sitter space," arXiv:hepth/0112166.

[5] I. R. Klebanov and E. Witten, "AdS/CFT correspondence and symmetry breaking," Nucl. Phys. B 556 (1999) 89 arXiv:hep-th/9905104.

[6] M. Flato and C. Fronsdal, Lett. Math. Phys. 2 (1978) 421; Phys. Lett. 97B (1980) 236; J. Math. Phys. 22 (1981) 1100; Phys. Lett. B172 (1986) 412.

[7] B. Binegar, C. Fronsdal and W. Heidenreich, "Conformal QED," J. Math. Phys. 24 (1983) 2828.

[8] S. S. Gubser, I. R. Klebanov and A. M. Polyakov, "Gauge theory correlators from noncritical string theory," Phys. Lett. B 428 (1998) 105 arXiv:hep-th/9802109. 
[9] E. Witten, "Anti-de Sitter space and holography," Adv. Theor. Math. Phys. 2 (1998) 253 [arXiv:hep-th/9802150].

[10] I. Bars and M. Günaydin, Commun. Math. Phys. 91 (1983) 31; M. Günaydin and S. J. Hyun, J. Math. Phys. 29 (1988) 2367; M. Günaydin and C. Saclioglu, Commun. Math. Phys., 87 (1982) 159; Phys. Lett. B108 (1982) 180.

[11] M. Günaydin, Oscillator Like Unitary Representations Of Noncompact Groups And Supergroups And Extended Supergravity Theories, Extended version presented at 11th Int. Colloq. on Group Theoretical Methods in Physics, Istanbul, Turkey, Aug 23-28, 1982. Published in Istanbul Grp.Th.Meth. (1982) 192; H. Nicolai, Representations Of Supersymmetry In Anti-De Sitter Space. Published in Trieste School (1984) 368; M. Duff, Int. J. Mod. Phys. A14 (1999) 815.

[12] S. Ferrara, "Algebraic Properties Of Extended Supergravity In De Sitter Space," Phys. Lett. B 69 (1977) 481. 999) 815.

[13] W. Nahm, "Supersymmetries And Their Representations," Nucl. Phys. B 135 (1978) 149.

[14] V. G. Kac, "Lie Superalgebras," Adv. Math. 26 (1977) 8.

[15] A. Galperin, E. Ivanov, S. Kalitsyn, V. Ogievetsky and E. Sokatchev, "Unconstrained N=2 Matter, Yang-Mills And Supergravity Theories In Harmonic Superspace," Class. Quant. Grav. 1 (1984) 469; "Unconstrained Off-Shell N=3 Supersymmetric Yang-Mills Theory," Class. Quant. Grav. 2 (1985) 155.

[16] P. S. Howe and G. G. Hartwell, "A Superspace Survey," Class. Quant. Grav. 12 (1995) 1823; “(N, p, q) harmonic superspace," Int. J. Mod. Phys. A 10 (1995) 3901 arXiv:hepth/9412147]; P. Heslop and P. S. Howe, "On harmonic superspaces and superconformal fields in four dimensions," Class. Quant. Grav. 17 (2000) 3743 arXiv:hep-th/0005135.

[17] A. S. Galperin, E. A. Ivanov, V. I. Ogievetsky and E. S. Sokatchev, "Harmonic Superspace," Cambridge, UK: Univ. Pr. (2001) 306 p.

[18] S. Ferrara and C. Fronsdal, "Gauge fields as composite boundary excitations," Phys. Lett. B 433 (1998) 19 arXiv:hep-th/9802126.

[19] M. Gunaydin and N. Marcus, "The Unitary Supermultiplet Of N=8 Conformal Superalgebra Involving Fields Of Spin $i=2$," Class. Quant. Grav. 2 (1985) L19.

[20] L. Andrianopoli, S. Ferrara, E. Sokatchev and B. Zupnik, "Shortening of primary operators in N-extended SCFT(4) and harmonic-superspace analyticity," Adv. Theor. Math. Phys. 3 (1999) 1149 arXiv:hep-th/9912007; S. Ferrara and E. Sokatchev, "Superconformal interpretation of BPS states in AdS geometries," Int. J. Theor. Phys. 40 (2001) 935 arXiv:hep-th/0005151]; "Conformal primaries of $\operatorname{OSp}(8 / 4, \mathrm{R})$ and BPS states in $\operatorname{AdS}(4)$," JHEP 0005 (2000) 038 arXiv:hep-th/0003051. 